TAUBE Karl A., Olmec Art at Dumbarton Oaks, Dumbarton Oaks Research Library and Collection, Washington, D.C., 2004, 228 p., bibl., index, ill., fig., photos, schéma, tabl.

\title{
Eric Taladoire
}

\section{(2) OpenEdition}

\section{Journals}

Édition électronique

URL : https://journals.openedition.org/jsa/3215

DOI : 10.4000/jsa.3215

ISSN : 1957-7842

Éditeur

Société des américanistes

Édition imprimée

Date de publication : 1 juin 2006

ISSN : 0037-9174

Référence électronique

Eric Taladoire, "TAUBE Karl A., Olmec Art at Dumbarton Oaks, Dumbarton Oaks Research Library and Collection, Washington, D.C., 2004, 228 p., bibl., index, ill., fig., photos, schéma, tabl. », Journal de la Société des américanistes [En ligne], 92-1 et 2 | 2006, mis en ligne le 06 avril 2007, consulté le 04 septembre 2022. URL : http://journals.openedition.org/jsa/3215 ; DOI : https://doi.org/10.4000/jsa. 3215

Ce document a été généré automatiquement le 4 septembre 2022

Tous droits réservés 


\title{
TAUBE Karl A., Olmec Art at Dumbarton Oaks, Dumbarton Oaks Research Library and Collection, Washington, D.C., 2004, 228 p., bibl., index, ill., fig., photos, schéma, tabl.
}

\author{
Eric Taladoire
}

1 Deuxième volume d'une nouvelle série de la Dumbarton Oaks, consacrée à ses propres collections (le premier, par Elizabeth Hill Boone, couvre les collections de l'aire andine), l'ouvrage de Karl Taube présente donc les 39 pièces olmèques de l'institution. Il n'est pas nécessaire de revenir ici sur l'origine de cette collection, puisque tous les spécialistes connaissent la fascination qu'exerçaient les jades olmèques sur Robert Woods Bliss, thème rappelé d'emblée dans la préface du livre (rédigée par Jeffrey Quilter) et dans l'introduction.

Plus qu'un simple catalogue, le livre est une étude fouillée, organisée en trois parties distinctes, mais très cohérentes. Une longue introduction de Karl Taube ( 47 pages) présente tout d'abord l'historique des recherches jusqu'aux travaux en cours, puis une synthèse des connaissances et de ses propres perspectives sur la civilisation olmèque. Vient ensuite le catalogue proprement dit et, enfin, une courte note (18 pages) sur l'origine des jades olmèques et les gisements du bassin du Motagua. Cette annexe trouve évidemment sa justification dans la nature même des objets, puisque les pièces en jadéite en forment la quasi-totalité. Pour ce qui touche au catalogue proprement dit, le cœur de l'ouvrage, il est remarquablement bien conçu. Chaque pièce est identifiée (origine, datation proposée, fiche technique, données bibliographiques), décrite et analysée, puis fait l'objet d'une étude iconographique et interprétative détaillée. Par ailleurs, chaque notice est illustrée d'une planche en couleur de la pièce, fréquemment accompagnée d'autres clichés en noir et blanc qui montrent l'objet sous des angles différents. Cette diversité de prises de vue est d'autant plus utile que trop de pièces souffrent justement 
de n'être présentées que sous l'angle principal, ce qui nuit à la compréhension d'objets par définition tridimensionnels. De plus, l'angle même selon lequel l'objet est considéré peut induire des différences d'interprétations. Ainsi en va-t-il, par exemple, pour un buste (Fragmentary figure, planche 16) qui, selon les auteurs, serait masculin ou féminin selon qu'il est vu de face ou de trois-quarts. L'inclusion de plusieurs photos trouve ainsi sa justification, car elles accompagnent véritablement le texte. Dans sa conception même, donc, ce catalogue est une réussite indéniable, ouvrage d'art autant qu'étude scientifique. Un autre de ses mérites provient justement de la réunion en un seul volume de toutes ces pièces, souvent connues. Elles ont pratiquement toutes été déjà illustrées, commentées ou présentées dans des expositions publiques. Mais les voir rassemblées ici traduit l'importance de la collection, comme elle est révélatrice des goûts et des préférences de celui qui en est à l'origine. Ce n'est pas le moindre apport de ce livre.

Cela posé, on ne peut pas oublier que l'ensemble des pièces est dépourvu de provenance. Or, si l'auteur lui-même mentionne (p. 207) l'existence de falsifications, attribuées à William Spratling, au Guerrero, en aucun cas il n'émet la moindre réserve sur l'authenticité des objets. Certes, la plupart ont été acquis à des époques anciennes où le faux olmèque était encore rare, mais tout de même est-il possible de poser franchement la question, ne serait-ce que pour les objets réputés provenir de Las Bocas, un site particulièrement mal connu, d'où seraient issues plusieurs autres pièces douteuses. De même, on peut rester dubitatif devant l'attribution de plusieurs pièces à l'art olmèque, par exemple les perles tubulaires (planche 37), ou le petit masque de la photo 38. Rien ne permet en réalité de trancher en faveur d'une appartenance établie et, comme le dit Taube lui-même, " tubular jade beads often are difficult to source and date » (p. 174). Dans ces conditions, une attribution de ces objets à d'autres civilisations est bel et bien envisageable, et l'on saisit mal pourquoi l'auteur choisit de les inclure dans son catalogue.

Il existe, par ailleurs, des préjugés qui affaiblissent le texte. L'auteur de la préface avait averti le lecteur: "Many of Taube's insights are new and intriguing, and some may be controversial... » (p.X). Cela dit, même sur des points plus fondamentaux que telle ou telle interprétation, il est permis d'exprimer un désaccord. La civilisation olmèque s'est développée durant près d'un millénaire (depuis 1500 jusqu'à environ 500 av. J.-C.). De plus, comme le souligne Taube, elle s'est enrichie d'apports extérieurs, voire antérieurs, comme ceux provenant du Soconusco ou de la côte Pacifique. Taube mentionne expressément, sur ce point, les travaux de Blake, Clark, Lowe ou Hill, pour ne citer que les plus récents. Enfin, le fond culturel olmèque a été transmis à des cultures postérieures et, là encore, Taube fait une place à part à des sites comme Tres Zapotes ou Cerro de las Mesas. Compte tenu de la durée du phénomène, il est peu concevable que ne se soit pas produite une évolution complexe, manifeste aussi bien dans le matériel archéologique que dans l'iconographie. Or, si l'auteur semble bien en être conscient, l'analyse iconographique proposée paraît ne pas en tenir compte. On se demande ainsi fréquemment sur quelles bases l'auteur avance, pour des objets sans contexte, un placement chronologique qui n'est aucunement démontré, mais seulement affirmé en fonction de similitudes plus ou moins convaincantes. Dans un autre registre, l'auteur balance, dans ses analyses, entre une interprétation des créatures olmèques en termes de forces naturelles ("divinities»), de panthéon de créatures structuré et de chamanisme. Avec près d'un millénaire d'existence, il est assez probable que la religion olmèque a subi des transformations et que des entités surnaturelles ont progressivement atteint le statut de véritables divinités. Encore doit-on rappeler que la discussion sur l'existence 
d'un panthéon structuré pour des civilisations postérieures reste d'actualité. En fait, il serait possible d'envisager les diverses interprétations mises en avant. Toutefois, ce qui gêne c'est qu'elles soient placées sur le même plan, voire appuyées sur les mêmes objets. Sans envisager d'évolution, Taube fausse les données et gomme la profondeur culturelle. Il introduit de plus des confusions dans l'esprit du lecteur qui peine à suivre le fil de sa pensée.

Plus contestable encore peut-être est l'attitude que Taube adopte en s'appuyant sur l'idée que les Olmèques constituent la culture mère de la Mésoamérique. D'autres chercheurs (Grove, Flannery et Marcus, Niederberger) ont émis des réserves à propos de cette théorie avec des arguments que l'on peut certes discuter, mais que Taube balaie d'un geste large. Au nom de l'unité culturelle mésoaméricaine, il s'autorise à effectuer des comparaisons sans tenir compte ni de la chronologie, ni du contexte. Il interprète ainsi des motifs en se référant aux Aztèques, aux Mayas, voire à des cultures dont on sait qu'elles ont échappé à toute influence olmèque, comme l'Occident du Mexique. Si parfois la démarche est malgré tout intéressante, dans d'autres cas, elle laisse le lecteur dubitatif, surtout lorsque l'auteur en arrive à des raisonnements circulaires. Pour illustrer ce point, on prendra l'exemple du Vieillard assis (planche 17). L'objet montre donc un personnage adulte, barbu, assis les jambes pliées. L'âge et le port de la barbe sont deux traits du Vieux Dieu, Huehueteotl, mais l'attitude diffère et d'autres attributs de ce dieu sont absents. Taube, ne prenant en compte que les similitudes, voit donc dans cette statuette une image du vieux dieu et la figurine devient l'ancêtre de Huehueteotl. Mais l'affaire devient nettement plus contestable quand Taube renverse la proposition: « Small greenstone carvings of Huehueteotl continued to be fashioned in Classic Mesoamerica». Du comparatisme, Taube est même tenté d'en faire avec d'autres cultures lointaines: il introduit ainsi le thème du Big Man de Mélanésie. Mais on peut aussi se demander s'il ne croit pas à une certaine diffusion entre les Olmèques et l'aire andine. De fait, les allusions à Chavin ou à d'autres cultures péruviennes contemporaines sont fréquentes, avec en filigrane l'idée d'une parenté. La note 4 (p. 7) est, à cet égard, particulièrement claire : « $A$ possible I shaped ball-court, strickingly similar to Mesoamerican examples, has recently been reported for coastal Peru, at the Initial Period site of Moxeke, dating from approximately 1600 to 1200 B.C. ». À la différence de Pozorski et Pozorski (dans « An I shaped ball-court form at Pampa de las Llamas-Moxeke, Peru », Latin American Antiquity, 6 (3), pp. 274-280, 1995), qui s'amusent d'une similitude superficielle de plan, mais soulignent une fonction probable très différente, Taube accorde à la ressemblance formelle beaucoup d'importance et $\mathrm{y}$ ajoute l'argument chronologique pour y voir une influence possible. Sur ce même sujet du jeu de balle, qui occupe dans l'introduction une place certaine, il est pourtant difficile d'accepter la position de Taube. Ce dernier considère en effet ce rituel comme un élément essentiel de la pensée cosmologique olmèque qu'il associe aux rites de fertilité, au Dieu Maïs, aux sacrifices d'enfants. Or, et il le dit lui-même, si un terrain du Formatif Ancien est connu à Paso de la Amada, sur la côte Pacifique du Chiapas, aucun terrain n'a encore été identifié avec certitude sur les sites de la zone nucléaire olmèque. Certes, des boules de caoutchouc ont été trouvées à El Manati, mais rien ne prouve qu'il s'agisse de balles, puisque le caoutchouc est connu pour la multiplicité de ses usages. Taube reprend, sans plus de preuve ni d'arguments, la vieille hypothèse selon laquelle les têtes colossales représenteraient des joueurs casqués et tente d'établir des liens iconographiques très ténus entre des objets tenus à la main par quelques personnages et les bâtons utilisés par les joueurs de la fresque du Tlalocan. En réalité, si l'on excepte de rares figurines, aucune donnée interne ne permet, à ce jour, d'affirmer l'existence du jeu chez les Olmèques. Dans 
ces conditions, l'hypothèse de l'importance du jeu chez les Olmèques n'est affaire que d'opinion personnelle. Un peu de la même façon, des pans entiers du texte ne sont que des affirmations fragiles et contestables. L'accumulation de ces à-peu-près, de ces hypothèses non argumentées affaiblit un travail qui a pourtant le mérite de rendre accessible un matériel insuffisamment connu. Cela nuit, hélas, à la qualité de l'ouvrage.

\section{AUTEURS}

ERIC TALADOIRE

université Paris I 